Canadian Oncology

Nursing Journal

Revue canadienne

de soins infirmiers

en oncologie

Volume 27, Issue 4 • Fall 2017

elSSN: 2368-8076 


\title{
Adaptation et utilisation du « modèle synergique » pour les patients hospitalisés à l'unité d'hématologie
}

\author{
par Yayra Amenudzie, Georgia Georgiou, Enoch Ho et Elizabeth O'Sullivan
}

\section{RÉSUMÉ}

Un projet pilote a été mis de l'avant par une unité de soins en hématologie et de greffes de cellules souches hématopoiétiques pour déterminer s'il est possible d'adapter le "modèle synergique » (Curley, 2007; 1998) à cette population de patients. Durant la phase 1, un outil de mesure des caractéristiques des patients portant sur la complexité, la stabilité, la prévisibilité et la participation aux soins a été développé et testé. Cet outil s'est révélé hautement valide et cohérent, et doté d'une validité conceptuelle et d'une concordance interévaluateurs modérée fort apparentes. Une évaluation de la compétence des infirmières a aussi été mise au point, ainsi que des procédures pour l'affectation des infirmières et des aides-soignants et un outil décisionnel pour la dotation. Les résultats de ce projet pilote démontrent que le modèle synergique peut être adapté à cette population et qu'il est possible de l'utiliser avec des patients hospitalisés.

\section{CONTEXTE ET BUT}

T'unité des hémopathies malignes et des greffes de cellules ـsouches hématopoïétiques de l'hôpital Juravinski a été transformée pour réunir deux équipes cliniques, de l'équipement et des services de soutien. Bien que cette transition se soit avérée une réussite à maints égards, de bonnes difficultés se sont posées pour l'affectation du personnel infirmier et des aides-soignants en raison des caractéristiques physiques de l'unité, de

\section{AU SUJET DES AUTEURES}

Yayra Amenudzie, inf. aut., M.Sc.inf., CON(C), infirmière en hématologie

Georgia Georgiou, B.A., M.Ed, gestionnaire clinique du programme de greffe de cellules souches

Enoch Ho, M.Pht., pht., acup., Bureau de l'amélioration de la qualité et de la valeur; hoenoch@HHSC.CA, Tél. : 905-521-2100, poste 42097

Elizabeth O'Sullivan, inf. aut., B.Sc.inf., M.Sc.inf., directrice, elizabethosullivan29@gmail.com

Auteures-ressources : Georgia Georgiou, B.A., M.Ed., Yayra Amenudzie, inf. aut., M.Sc.inf., Unité d'hématologie Ron et Nancy Clark, Centre hospitalier et de cancérologie Juravinski, Centre des sciences de la santé d'Hamilton, 699, rue Concession, Hamilton (Ontario) L8V 5C2

905-521-2100, poste 43910

Courriel : georgg@hhsc.ca ou amenudzie@hhsc.ca

DOI: $10.5737 / 23688076274343347$ l'acuité élevée des soins aux patients, du grand nombre d'infirmières novices, des différentes pratiques infirmières entre les deux équipes cliniques et des ressources d'aides-soignants limitées (un aide-soignant pour 39 patients par quart de travail). Il y avait des incohérences dans les décisions d'affectation d'un quart de travail à un autre, ce qui entraînait des frictions entre les membres de l'équipe, des plaintes sur la charge de travail, et des affectations dangereuses pouvant exposer à des risques tant les patients que le personnel. La charge de travail élevée des infirmières, l'acuité des soins aux patients et les compétences infirmières très hétérogènes perturbaient l'équipe, le moral, la qualité des soins aux patients, la satisfaction des patients et du personnel, le recrutement et la fidélisation du personnel. L'équipe a identifié le besoin d'améliorer l'environnement de travail en élaborant un modèle décisionnel objectif permettant une affectation qui tienne compte non seulement de l'acuité des soins aux patients, mais aussi de la compétence de l'infirmière.

L'instauration d'un ratio infirmière-patient obligatoire ou de systèmes de classification des patients ont été proposés comme approches pour garantir des affectations adéquates du personnel infirmier. Toutefois, une analyse documentaire a permis de trouver qu'une méthode d'affectation qui inclut directement l'avis des infirmières des unités dans les décisions d'affectation quotidienne est justifiée, car de nombreuses variables ont une incidence sur les décisions d'affectation quotidienne (Hertel, 2012).

Cette observation a mené notre équipe à piloter le projet de modèle synergique pour diverses raisons. D'abord, le modèle synergique est un modèle pratique professionnel géré par les infirmières, ce qui remet entre leurs mains le contrôle de leur pratique et de leur environnement de travail (MacPhee, 2011). Ensuite, le modèle porte sur les besoins des patients pour déterminer les compétences requises chez les infirmières et les exigences sur le plan des ressources. Bien qu'il soit né en milieu d'hospitalisation où la gravité est élevée et aiguë, il a été utilisé dans divers milieux et à de multiples fins. Des travaux de recherches publiés indiquent que le modèle a le potentiel de transformer la façon dont les prestataires de soins pensent aux patients et à la méthode pour procéder à l'affectation auprès des patients. Il fournit aux prestataires de soins un moyen pour déterminer les besoins de leurs patients et cibler les soignants les mieux appropriés pour répondre à ces besoins, afin d'obtenir les meilleurs résultats possible pour le patient. Bien que le modèle synergique se soit avéré bénéfique dans divers milieux de soins de santé comportant diverses populations de patients (Carter et Burnette, 2011; Pope, 2002; Gralton et Brett, 2012; Kaplow et Reed, 2008), nous n'avons pu en trouver d'application dans une unité d'hémopathies malignes et de greffes de cellules souches hématopoïétiques au Canada.

Le but de ce projet pilote était d'examiner s'il était possible d'adapter le modèle synergique pour l'affectation des 
infirmières dans une population de patients en hématologie/ oncologie, et d'évaluer son utilité et son impact. Cet article porte sur la première phase du projet pilote qui consistait à répondre à trois questions : Peut-on adapter le modèle synergique à une population de patients en hématologie? Est-il possible d'implanter ce modèle dans un milieu d'hospitalisation où l'acuité des soins est élevée? Quels défis rencontre-t-on et quelles stratégies facilitent la réussite de l'implantation? La deuxième phase du projet pilote, décrite plus loin, implique une évaluation de l'utilité du modèle et de son impact sur la prestation des soins et sur la pratique.

\section{MÉTHODOLOGIE}

Un groupe de travail sur la synergie a été formé pour diriger le projet pilote. Ce groupe de travail regroupait divers intervenants (personnel infirmier [cinq infirmières autorisées et une infirmière praticienne], aide-soignant, responsable de la formation clinique de l'unité, gestionnaire clinique, direction du programme, spécialiste en qualité, chef de la pratique interprofessionnelle), en plus de profiter de contributions ponctuelles de médecins et du parrainage de la vice-présidente de la pratique interprofessionnelle et directrice des soins infirmiers. Du financement provenant de deux subventions a permis à une infirmière de première ligne de consacrer du temps pour diriger le projet et l'encadrer. Le Guide provincial du projet sur la charge de travail des infirmières, qui décrit l'utilisation du modèle synergique par la British Columbia Nurse's Union (BCNU), a aussi servi de guide (BCNU, 2010).

Cette étude repose aussi sur une approche de recherche-action participative (RAP) utilisant des méthodes aussi bien qualitatives que quantitatives. Le groupe de travail sur la synergie susmentionné a participé activement au développement de l'outil et à son implantation. Des réunions mensuelles ont été tenues avec ce groupe principal pour analyser les données recueillies dans les entrevues, les groupes de discussion et les sondages. Un entretien de finalisation a eu lieu avec la responsable d'unité ayant dirigé l'implantation. Des sondages ont été réalisés auprès de 568 infirmières et de 73 chefs d'unité, puis analysés au moyen de mesures statistiques pour en vérifier la fiabilité, la validité, la cohérence et la portée. Des membres du personnel ont été invités à remplir un sondage sur l'environnement de travail avant le démarrage du projet pilote. Des thèmes communs ont été dégagés des groupes de discussion, des sondages et des entrevues.

La section ci-après précise la méthodologie utilisée pour 1) évaluer l'état de l'environnement de travail, 2) développer et valider l'outil des caractéristiques du patient pour notre population de patients, 3) mettre au point une évaluation de la compétence des infirmières en oncologie se spécialisant en hématologie et en greffe de cellules souches, 4) élaborer des directives sur l'affectation et 5) évaluer la possibilité d'implanter les outils et les procédés.

\section{Développement de l'instrument : outils sur les caractéristiques d'un patient}

Notre analyse environnementale a mis en lumière l'importance de développer des outils et des procédures à la fois simples et efficaces, utilisables dans la pratique quotidienne.
Le groupe de travail a utilisé la méthode socratique, soit une approche décisionnelle fondée sur le consensus, pour produire des marqueurs permettant de définir chacune des huit caractéristiques des patients en hématologie. Le groupe a révisé chaque marqueur et signalé ceux relevés dans plus d'une caractéristique. Ces marqueurs ont ensuite été positionnés en fonction de la caractéristique la plus appropriée pour le patient, puis les «meilleures » caractéristiques paraissant refléter les besoins du patient ont finalement été sélectionnées. Pour simplifier l'outil, le groupe de travail a choisi d'utiliser une échelle de notation en trois points plutôt qu'une échelle en cinq points, le niveau 1 représentant le niveau le plus aigu et le niveau 3, le moins aigu. Enfin, le groupe de travail a aussi choisi de consigner chaque résultat individuel pour chaque caractéristique plutôt que de calculer un score moyen tel que le proposait le modèle original.

Pour évaluer la validité apparente de l'outil, trois experts cliniques (hématologues) l'ont passé en revue et ont fourni une rétroaction sur l'efficacité du positionnement de chaque marqueur par rapport à la caractéristique du patient, ainsi que vérifié si des marqueurs ou des caractéristiques semblaient manquer ou paraissaient inutiles. Les médecins n'ont joué aucun rôle dans le développement de l'outil et ne connaissaient pas le modèle synergique. L'outil a été mis en pratique dans des études de cas réelles pour évaluer plus avant sa validité apparente.

Pour tester la validité conceptuelle de l'outil, on a demandé à deux hématologues ne connaissant pas le modèle synergique d'évaluer les patients en fonction de chaque caractéristique durant leurs visites, à l'aide d'une échelle en cinq points. Ils ont établi leur note sur l'évaluation courante du malade et leur jugement clinique. L'infirmière spécialisée a, au même moment, évalué ces mêmes patients en parallèle des médecins. Le médecin et l'infirmière n'avaient pas accès à leurs résultats respectifs et ces derniers ont été comparés au moyen du test de corrélation Pearson.

Enfin, la cohérence interne et la fiabilité interévaluateurs de l'outil ont aussi été déterminées au moyen du test alpha de Cronbach et du test de corrélation intraclasses, respectivement. La fiabilité interévaluateurs a été testée indépendamment par trois infirmières spécialisées en hématologie ayant évalué les mêmes patients sans avoir accès à leurs résultats respectifs.

\section{Développement de l'instrument : évaluation de la compétence des infirmières}

Une évaluation de la compétence des infirmières a été élaborée sur la base du modèle Benner (1984), des normes de l'Association canadienne des infirmières en oncologie (2002) et des caractéristiques précisées dans le modèle synergique (Curley, 2007). Toutes les infirmières en hématologie ont rempli l'auto-évaluation et ont été classées comme novice, débutante avancée, compétente, aguerrie ou experte.

\section{Établissement de procédures : directives pour l'affectation et la dotation}

Il n'existait aucune directive pour l'affectation des infirmières dans notre unité, qui en avait besoin. Ces directives devaient préciser le type de patients (niveau d'acuité) et le nombre de patients auquel une infirmière pouvait être affectée 
en fonction de sa compétence. Comme l'acuité des soins aux patients en hématologie fluctue et que le besoin de surveillance et d'intervention des infirmières est élevé, il était évident qu'il y aurait des moments où des ressources additionnelles seraient requises pour répondre aux directives d'affectation. L'approbation et le soutien de la haute direction ont été obtenus pour établir des critères pour trouver d'autres infirmières et aides-soignants en tenant compte de l'acuité des soins prodigués aux patients.

Pour établir des directives d'affectation, on a demandé aux infirmières de remplir un sondage à chaque quart de travail durant trois semaines pour juger du rythme de travail qu'elles avaient pu avoir : a) rythme idéal permettant d'aller au-delà du devoir, b) occupé, mais gérable, c) occupé et difficilement gérable, ou d) ingérable et non sécuritaire. On leur a aussi demandé d'indiquer si les notes d'acuité reflétaient l'acuité réelle des soins à prodiguer aux patients et de signaler leur niveau de compétence comme infirmière. Une procédure semblable a été utilisée pour élaborer des directives de dotation pour l'affectation des aides-soignants. Ces données ont ensuite été utilisées pour rédiger les directives pour l'affectation et pour la définition des seuils de dotation supplémentaire.

Deux groupes de discussion ont donné leur avis sur la façon de concevoir les procédures opérationnelles quotidiennes permettant d'évaluer les patients, de présenter et mettre à jour les notes attribuées, de procéder aux affectations et de demander des ressources additionnelles. Léchantillon pour les deux groupes de discussion avait été choisi à dessein et regroupait des infirmières de chaque niveau. La formation du personnel sur les outils et procédures s'est faite pendant des journées de formation et lors de rencontres individuelles. Il a été décidé que, pendant le projet pilote, les patients seraient notés seulement durant les quarts de travail de jour pour veiller à ce que le soutien nécessaire soit en place et faciliter la réussite de l'implantation.

\section{Méthodes d'évaluation de l'applicabilité du modèle synergique}

Les mesures suivantes ont été retenues pour déterminer si les outils et procédures proposés pourraient être appliqués : a) le nombre de fois en pourcentage où les infirmières ont rempli l'outil sur l'acuité des soins à prodiguer aux patients, b) le nombre de fois en pourcentage où les notes d'acuité et les compétences du personnel ont été utilisées pour procéder à l'affectation des infirmières, c) la fréquence à laquelle on a répondu aux critères d'augmentation des niveaux de dotation, et d) les facteurs limitants et facilitants liés à l'implantation. Ces données ont été recueillies pendant 73 quarts de travail de jour, du 13 janvier au 31 mars 2014, grâce à : un sondage rempli quotidiennement par des infirmières; un sondage quotidien au chef d'unité; des groupes de discussion; et une entrevue individuelle avec le chef d'unité principal.

\section{RÉSULTATS}

On a recueilli et analysé au total 568 sondages d'infirmières et 73 sondages de chefs d'unité, des commentaires de deux groupes de discussions et une entrevue avec le chef d'unité principal. Les résultats sont discutés en fonction de chacune des questions du projet pilote.
Question 1 : Peut-on adapter le modèle synergique aux patients en hématologie?

Un outil montrant l'acuité des soins à prodiguer aux patients en hématologie a été développé pour notre population de patients. Il comporte quatre caractéristiques : la participation aux soins, la prévisibilité, la complexité et la stabilité (voir Figure 1).

Les quatre caractéristiques et les marqueurs pour chacune ont obtenu le consensus des experts en hématologie, ce qui dénote une forte validité apparente. L'analyse des données issues du sondage quotidien aux infirmières indique que $94 \%$ des infirmières trouvaient que la note synergique reflétait bien l'acuité des soins à prodiguer au patient. Les résultats au test de cohérence interne ont indiqué un alpha de Cronbach supérieur à 0,87 pour les quatre caractéristiques (complexité $=0,89$; stabilité $=0,95$; participation aux soins $=0,89$; prévisibilité $=0,87$ ). Cela donne à penser que tous les éléments mesurent aussi bien l'objet de la recherche tout en apportant quelque chose d'unique.

L'outil a aussi montré qu'il était bon et valide sur le plan conceptuel. Les coefficients de corrélation pour chaque domaine allaient comme suit : complexité $=0,72$; prévisibilité $=0,77$; participation aux soins $=0,75$; stabilité $=0,54$. Les résultats du test de fiabilité interévaluateurs de l'outil utilisant la corrélation intraclasses ont été plus élevés que 0,70 pour toutes les caractéristiques, ce qui montre un accord important, parfois presque parfait, entre les évaluateurs (complexité $=0,81$; stabilité $=0,92$; participation aux soins $=0,77$; prévisibilité $=0,71$ ).

\section{Question 2 : Est-il possible d'appliquer ce modèle et les procédures?}

La plupart du temps, les outils et procédures ont été appliqués de manière uniforme. Les infirmières autorisées ont utilisé l'outil synergique sur les caractéristiques des patients pour noter les patients dans une proportion de quarts de travail évaluée à $77 \%$ et le chef d'unité principal a utilisé les résultats pour procéder à l'affectation à tous les quarts de travail (100\%). Il a fallu des aides-soignants supplémentaires pour 27 quarts de travail, ainsi qu'une infirmière autorisée supplémentaire pour 23 quarts de travail. Cependant, il n'a pas toujours été possible d'augmenter le personnel au moment requis par manque d'effectifs disponibles ou en raison des frais qu'auraient occasionnés les heures supplémentaires.

\section{DISCUSSION}

\section{Limites du modèle}

Le modèle synergique est conçu pour mesurer l'acuité des soins au patient et non la charge de travail. Il faut néanmoins tenir compte de cette charge de travail au moment de procéder à l'affectation. L'inconvénient de faire passer l'échelle d'acuité de cinq à trois points est qu'il devient plus difficile de faire ressortir les différences affectant la charge de travail. À titre d'exemple, deux patients pourraient obtenir les mêmes notes, mais exiger différents soins infirmiers plus ou moins longs. L'outil pourrait être amélioré pour qu'il tienne compte des interventions qui exigent un suivi plus rapproché ou davantage de soins infirmiers.

Bien que le modèle synergique permette de procéder à l'affectation des infirmières de manière plus objective, la procédure prend un certain temps en plus d'être assez complexe et 
Figure 1 : Outil évaluant l'acuité de 4 caractéristiques des soins aux patients en hématologie

\section{ÉVALUATION EN 4 CARACTÉRISTIQUES DE L'ACUITÉ DES SOINS PRODIGUÉS AUX PATIENTS} MODÈLE SYNERGIQUE ADAPTÉ À L'HÉMATOLOGIE (Ne pas reproduire sans permission)

Nom Salle Date

Consultez la section « Définitions » pour les termes avec astérisque (Définitions accessibles sur disque partagé ou formulaire laminé sur les tableaux en liège aux postes) Niveau 1 -> Acuité élevée Niveau 2 -> Acuité modérée Niveau 3 -> Acuité moindre

* Nous notons les patients en fonction de leurs besoins actuels, sauf si «prochain quart de travail » est explicitement écrit.

* Patients en soins palliatifs* : inutile de les noter dans la section stabilité.

\section{A-STABILITÉ}

\begin{tabular}{|c|c|c|}
\hline$\square$ Saigne beaucoup* & $\begin{array}{l}\square \text { Hg entre } \mathbf{8 0 - 8 5} \text { OU } \\
\text { plaquettes inférieures à } 25 \\
\text { ET aucun don de RCC ou } \\
\text { de Plts durant ce quart } \\
\square \text { Réfractaire aux plaquettes }\end{array}$ & $\begin{array}{l}\square \text { HG au-dessus de } 85 \text { OU } \\
\text { plaquettes à } 25 \text { ou plus } \\
\square \text { Hb }<\mathbf{8 0} \text { ou Plts }<10^{*} \\
\text { et don de RCC ou de } \\
\text { plaquettes durant ce quart } \\
\text { avec pt non réfractaire aux } \\
\text { plaquettes }\end{array}$ \\
\hline $\begin{array}{l}\text { J Plus de } 2 \text { valeurs de labo* } \\
\text { déséquilibrées exigeant des } \\
\text { interventions autres que } \\
\text { des transfusions de produits } \\
\text { sanguins }\end{array}$ & $\begin{array}{l}\text { D } 1 \text { ou } 2 \text { valeurs de labo* } \\
\text { déséquilibrées exigeant des } \\
\text { interventions autres que } \\
\text { des transfusions de produits } \\
\text { sanguins }\end{array}$ & $\begin{array}{l}\square \text { Valeurs de labo* stables } \\
\text { ou } \\
\square \text { Valeur de labo déséquili- } \\
\text { brée mais n'exigeant aucune } \\
\text { intervention }\end{array}$ \\
\hline $\begin{array}{l}\text { Neutropénie et fièvre } \\
\text { supérieure à } 38^{\circ}\end{array}$ & $\begin{array}{l}\square \text { Neutropénie; aucune } \\
\text { fièvre } \\
\square \text { Pas de neutropénie; } \\
\text { fièvre }\end{array}$ & $\checkmark \mathrm{Ni}$ neutropénie, ni fièvre \\
\hline $\begin{array}{l}\square \text { Apparition* de signes } \\
\text { vitaux instables exigeant une } \\
\text { intervention (signes vitaux } \\
\text { hors norme chez ce pt; excl. } \\
\text { la fièvre). }\end{array}$ & $\begin{array}{l}\square \text { Signes vitaux stables avec } \\
\text { interventions }\left(\mathrm{O}_{2} \text { inclus }\right) \\
\square \text { Signes vitaux instables } \\
\text { avec surveillance continue et } \\
\text { intervention }\end{array}$ & $\begin{array}{l}\text { Signes vitaux stables ou } \\
\text { normaux chez ce patient - } \\
\text { aucune intervention requise }\end{array}$ \\
\hline $\begin{array}{l}\text { MVO ou surcharge } \\
\text { liquidienne* exigeant } \\
2 \text { interventions ou > }\end{array}$ & $\begin{array}{l}\text { Surcharge liquidienne } \\
\text { exigeant une intervention }\end{array}$ & $\begin{array}{l}\text { Aucune surcharge } \\
\text { liquidienne OU aucune } \\
\text { intervention requise }\end{array}$ \\
\hline $\begin{array}{l}\square \text { Toutes nouvelles* } \\
\text { urgences hématologiques } \\
\text { ou oncologiques* durant les } \\
24 \text { premières heures } \mathbf{O U} \\
\square \text { Aucune réponse au traite- } \\
\text { ment requis par l'urgence }\end{array}$ & $\begin{array}{l}\text { DToutes urgences hémato- } \\
\text { logiques ou oncologiques* } \\
\text { présentant une amélioration } \\
\text { durant le traitement }\end{array}$ & $\begin{array}{l}\text { Aucune urgence hémato- } \\
\text { logique ou oncologique* }\end{array}$ \\
\hline $\begin{array}{l}\square \text { Cognition altérée } \\
\text { exigeant } 1: 1\end{array}$ & $\begin{array}{l}\square \text { Cognition altérée, mais } \\
\text { aisément contournable }\end{array}$ & $\square$ Alerte et orienté $x 3$ \\
\hline
\end{tabular}

\section{C-PRÉVISIBILITÉ}

\begin{tabular}{|c|c|c|}
\hline $\begin{array}{l}\text { Daladie incontrôlée, non } \\
\text { gérée ou exacerbée par le } \\
\text { traitement actif retenu pour } \\
\text { le soin } \\
\square \text { Diagnostic nouveau/ } \\
\text { inconnu }\end{array}$ & $\begin{array}{l}\square \text { Amélioration du Pt en } \\
\text { traitement } \\
\square \text { Symptômes du pt gérés } \\
\text { et réponse au traitement } \\
\text { (chimio ou transplantation) }\end{array}$ & $\begin{array}{l}\square \text { Maladie bien contrôlée } \\
\text { ou gérée } \\
\square \text { Palliatif : fin de stade } \\
\text { congruent avec les objectifs } \\
\text { du traitement }\end{array}$ \\
\hline ] Aucun congé prévu & $\begin{array}{l}\square \text { Recevra son congé d'ici } \\
72 \text { hres }\end{array}$ & $\begin{array}{l}\text { Recevra son congé d'ici } \\
24 \text { hres }\end{array}$ \\
\hline
\end{tabular}

B-COMPLEXITÉ*

\begin{tabular}{|c|c|c|}
\hline $\begin{array}{l}\square 3 \text { perfusions IV ou plus en } \\
\text { continu } \\
\square>\text { de } 6 \text { médications IV prévues }\end{array}$ & $\begin{array}{l}\square 2 \text { à } 6 \text { médications IV } \\
\text { prévues }\end{array}$ & $\begin{array}{l}\square 1 \text { perfusion IV ou moins } \\
\square 0 \text { - } 1 \text { médication IV prévue }\end{array}$ \\
\hline $\begin{array}{l}\text { Au prochain quart le pt reçoit : } \\
\square \text { Chimio avec prémédication et } \\
\text { surveillance étroite * } \\
\square \text { Chimio en IV } \\
\square \text { Agent/protocole nouveau } \\
\square>\text { de } 2 \text { chimio par quart }\end{array}$ & $\begin{array}{l}\text { Au prochain quart le pt } \\
\text { reçoit : } \\
\square \text { Une ou deux chimio en IV } \\
\text { le même jour } \\
\square \text { Chimio IT } \\
\square \text { Chimio sous-cutanée* }\end{array}$ & $\begin{array}{l}\text { Au prochain quart le pt } \\
\text { reçoit : } \\
\square \text { Aucune chimio IV } \\
\square \text { Chimio PO }\end{array}$ \\
\hline $\begin{array}{l}\square 4 \text { produits sanguins ou plus } \\
\square \text { Produit sanguin exigeant une } \\
\text { surveillance étroite (p. ex. IVIG) } \\
\square \text { Jour } 0 \text { de l'Allo ou de } \\
\text { l'Auto-SCT }\end{array}$ & $\begin{array}{l}\square 2 \text { à } 3 \text { produits sanguins en } \\
\text { fonction des niveaux de Hg et } \\
\text { de plaquettes }\end{array}$ & $\begin{array}{l}\text { Aucun produit sanguin ou } \\
\text { un seul produit sanguin }\end{array}$ \\
\hline $\begin{array}{l}\text { Douleur exigeant une infusion } \\
\text { de narcotique en continu*, IV } \\
\text { ou inj. SC }\end{array}$ & $\begin{array}{l}\square \text { Douleur contrôlée selon } \\
\text { un horaire et/ou médications } \\
\text { orales innovatrices }\end{array}$ & $\square$ Aucune douleur \\
\hline $\begin{array}{l}\text { Symptômes GI incontrôlés* } \\
\text { exigeant d'autres interventions }\end{array}$ & $\begin{array}{l}\square \text { Symptômes } \mathrm{GI}^{*} \text { contrôlés } \\
\text { avec horaire d'interventions }\end{array}$ & $\begin{array}{l}\square \text { Aucun symptôme et au- } \\
\text { cune intervention requise }\end{array}$ \\
\hline $\begin{array}{l}\text { Alimentation NG/PEG en } \\
\text { continu ou intermittente }\end{array}$ & $\square \mathrm{TPN}$ & $\begin{array}{l}\square \text { Alimentation PO partielle/ } \\
\text { adéquate } \\
\square \text { NPO selon le type de soins }\end{array}$ \\
\hline$\square$ Pt sous dialyse ou CBI & $\begin{array}{l}\square \text { Scan de la vessie prévue } \\
\square \text { Sonde à demeure ou } \\
\text { intermittente }\end{array}$ & $\square$ Miction sans intervention \\
\hline $\begin{array}{l}\square \text { Plaies de stade } 3 \text { et } 4 \\
\square \text { Quart du lendemain : Pt } \\
\text { requiert des soins importants de } \\
\text { la peau et des plaies* }\end{array}$ & $\begin{array}{l}\square \text { Plaies de stade } 2 \\
\square \text { Quart du lendemain : Pt } \\
\text { requiert des soins modérés } \\
\text { de la peau et des plaies* }\end{array}$ & $\begin{array}{l}\square \text { Plaie de stade } 1 \text { requérant } \\
\text { une assistance minimale ou } \\
\text { aucune plaie }\end{array}$ \\
\hline $\begin{array}{l}\square \text { Pt et famille en détresse } \\
\square \text { Habiletés d'adaptation } \\
\text { réduites et/ou pt a un système de } \\
\text { soutien inadéquat } \\
\square \text { Barrières linguistiques/ } \\
\text { culturelles importantes exigeant } \\
\text { des interventions }\end{array}$ & $\begin{array}{l}\square \text { Soins de soutien émotion- } \\
\text { nel modéré requis } \\
\square \text { Habiletés d'adaptation et } \\
\text { système de soutien adéquat } \\
\square \text { Quelques obstacles à la } \\
\text { communication }\end{array}$ & $\begin{array}{l}\square \text { Pt et famille sans détresse } \\
\square \text { Bon système de soutien } \\
\square \text { Aucune barrière communi- } \\
\text { cationnelle }\end{array}$ \\
\hline $\begin{array}{l}\text { Antécédents de comorbidité } \\
\text { avec symptômes incontrôlés } \\
\text { exigeant des interventions et/ou } \\
\text { de longues consultations }\end{array}$ & $\begin{array}{l}\text { Antécédents de comor- } \\
\text { bidité }{ }^{*} \text { avec symptômes } \\
\text { incontrôlés exigeant des } \\
\text { interventions }\end{array}$ & $\begin{array}{l}\square \text { Aucune comorbidité } \\
\text { ou comorbidité n'exigeant } \\
\text { aucune intervention }\end{array}$ \\
\hline $\begin{array}{l}\square \text { Procédure au cours des } \\
24 \text { prochaines heures exigeant } \\
\text { une surveillance au Q15 min }\end{array}$ & $\begin{array}{l}\text { Procédure au cours } \\
\text { des } 24 \text { prochaines heures } \\
\text { exigeant une surveillance } \\
\text { au Q2hrs }\end{array}$ & $\begin{array}{l}\square \text { Aucune procédure avec } \\
\text { surveillance au cours des } \\
24 \text { prochaines heures }\end{array}$ \\
\hline $\begin{array}{l}\square \text { UPH Q3HRS } \\
\square \text { CBG QID }\end{array}$ & $\begin{array}{l}\square \text { Analyse sanguine BID } \\
\square \text { CBG BID }\end{array}$ & $\begin{array}{l}\text { Analyses sanguines } \\
\text { quotidiennes }\end{array}$ \\
\hline
\end{tabular}

D-PARTICIPATION AUX SOINS*

\begin{tabular}{|l|l|l|}
\hline \multicolumn{2}{|l|}{} \\
\hline $\begin{array}{l}\square \text { Pt incapable d'accomplir } \\
\text { des AVQ }\end{array}$ & $\begin{array}{l}\square \text { Requiert de l'aide pour } \\
\text { les AVQ }\end{array}$ & $\square$ Accomplit seul les AVQ \\
\hline $\begin{array}{l}\square \text { Pt dépend du personnel } \\
\text { et d'appareils d'aide* pour } \\
\text { la toilette }\end{array}$ & $\begin{array}{l}\square \text { Utilise un appareil d'aide } \\
\text { pour se toiletter seul }\end{array}$ & $\begin{array}{l}\square \text { Peut se toiletter seul sans } \\
\text { l'aide d'appareils d'aide }\end{array}$ \\
\hline $\begin{array}{l}\square \text { Reste au lit, au fauteuil ou } \\
\text { immobile; aide de 2 personnes }\end{array}$ & $\begin{array}{l}\square \text { Est aidé par 1 personne } \\
\text { pour se déplacer }\end{array}$ & $\begin{array}{l}\square \text { Peut se déplacer seul (avec } \\
\text { déambulateur) }\end{array}$ \\
\hline $\begin{array}{l}\square \text { Dépend de soignants pour } \\
\text { être retourné ou repositionné }\end{array}$ & $\begin{array}{l}\square \text { Pt a besoin d'aide pour se } \\
\text { tourner et se repositionner }\end{array}$ & $\square$ Pt peut se repositionner seul \\
\hline \begin{tabular}{l}
$\square$ Isolement exigeant EPI \\
\hline
\end{tabular} & $\square$ Isolement inversé & $\square$ Aucun isolement \\
\hline
\end{tabular}


intimidante. Il faut en effet faire la synthèse de quatre notes pour 39 patients et tenir compte d'autres facteurs (continuité de l'affectation, aspects géographiques, etc.). La présence d'un chef d'unité principal durant 6 à 8 mois a donné l'occasion d'acquérir amplement de pratique. Il faut aussi une formation adéquate pour permettre aux infirmières de gagner en confiance, afin de procéder aux affectations en fonction des notes synergiques.

Atteindre la conformité avec des notes établies quotidiennement et mettre à jour ces notes lorsque l'acuité change exige un renforcement constant rendu possible par la mise en place de bonnes indications et structures de responsabilisation. Pour bien apparier les besoins des patients et les compétences des infirmières, il est nécessaire d'avoir à chaque quart de travail un éventail des compétences infirmières et un échantillon suffisamment diversifié d'infirmières compétentes, expertes et expérimentées. Lorsque le nombre de novices et de débutantes avancées est disproportionné au cours d'une rotation, il devient difficile de répondre aux directives d'affectation. Cela dit, le modèle peut aider à nourrir les décisions sur la dotation, à configurer de manière optimale la rotation principale et à planifier le développement professionnel du personnel infirmier. Enfin, s'il est prévu d'utiliser le modèle pour procéder à des ajustements d'affectation, il faut d'abord obtenir le soutien de la direction et un budget, et aussi avoir accès à un bassin de travailleurs à temps partiel assez grand pour éviter les coûts inhérents aux heures supplémentaires.

\section{Catalyseurs de la mise en œuvre}

Les réponses des groupes de discussion, des sondages et des entrevues pointaient vers plusieurs éléments ayant facilité le succès de l'implantation : a) le soutien et le parrainage de l'équipe de haute direction de l'hôpital; b) une gestion de projet solidement menée et un plan clair pour ne pas dérailler et respecter le budget; c) un accès à du financement permettant à une infirmière de première ligne de mener le projet pilote et de s'adjoindre du personnel de première ligne; d) la présence d'un chef d'unité principal doté d'un fort leadership; e) un accès à des qualiticiens, à des chefs praticiens et à des chercheurs expérimentés servant de guides et de mentors; f) un accès à du financement pour soutenir la formation du

\section{RÉFÉRENCES}

Benner, P. (1984). From novice to expert: Excellence and power in clinical nursing practice. Menlo Park: Addison-Wesley.

British Columbia Nurses Union. (2010). Provincial nursing workload project resource toolkit for teams: Nursing workload and staffing plan processes. Retrieved from https://www.bcnu.org/Safe-workplace/ DefendProfessionalPractice/Documents/pnwp_resource_toolkit. pdf

Canadian Association of Nurses in Oncology. (2017). Rationale for Standards of care. Retrieved from http://www.cano-acio.ca/page/ standards_of_care

Canadian Association of Nurses in Oncology. (2002). Practice standards and competencies. Retrieved from http://www.cano-acio.ca/conep.

Carter, K.F., \& Burnette, H.D. (2011). Creating patient-nurse synergy on a medical-surgical unit. Medsurg Nursing, 20, 249-254.

Curley, M.A. (Ed.). (2007). Synergy: The unique relationship between nurses and patients. Indianapolis: Sigma Theta Tau International.

Curley, M.A. (1998). Patient-nurse synergy: Optimizing patient's outcomes. American Journal of Critical Care, 7, 64-72. personnel sur les nouveaux outils et procédures; g) une vérification fréquente des données pour garantir leur intégrité et; h) la mise en place d'un processus pour mettre régulièrement à jour les compétences des infirmières autorisées.

\section{CONCLUSION}

Les résultats de notre projet pilote confirment que le modèle synergique peut être adapté à différentes populations de patients, comme le rapportaient d'autres chercheurs (MacPhee et al., 2011; Kaplow et Reed, 2008, Kerfoot et al., 2006, MacPhee et al., 2010). L'application du modèle à une unité d'hématologie a constitué un travail pionnier, mais l'outil et la méthodologie qui en ont émergé pourront être utilisés pour d'autres populations de patients. Les limites et les difficultés rencontrées peuvent être dépassées par diverses stratégies dont il a déjà été question. La nature flexible du modèle convient bien à la fluctuation de l'acuité et des besoins des patients en hématologie et des greffés. Il permet aux administrateurs de soins de santé de prendre sur le vif des décisions plus éclairées et efficaces quant à l'utilisation et à l'allocation des ressources en réponse aux changements d'acuité des soins.

Au final, comme l'ACIO le soutient (2017), « l'acuité et la complexité des besoins des personnes atteintes de cancer exigent des infirmières qu'elles possèdent un champ de connaissances élargies ». Il est, par conséquent, important que les besoins des patients comme les compétences des infirmières soient mesurables afin de pouvoir les apparier au mieux et ainsi optimiser les soins offerts. L'outil développé sur l'acuité des soins aux patients en hématologie et l'outil sur les compétences des infirmières permettront aux unités d'oncologie de mieux faire correspondre les besoins des patients avec les compétences des infirmières offrant des soins.

\section{REMERCIEMENTS}

Nous tenons à remercier notre équipe de projet, le personnel et les médecins de l'unité de soins en hématologie et de greffe de cellules souches hématopoiétiques, la Fondation du centre de cancérologie Juravinski et F. Hoffmann-La Roche Ltd pour le financement et le soutien durant ce projet pilote.

Gralton, K.S. \& Brett, S.A. (2012). Integrating the synergy model for patient care at Children's Hospital in Wisconsin. Journal of Pediatric Nursing, 27, 7481.

Hertel, R. (2012). Regulating patient staffing: A complex issue. MedSurg Matters, 21(1), 3-7.

Kaplow, R., \& Reed, K. (2008). The AACN synergy model for patient care: A nursing model as a force of magnetism. Nursing Economics, 26, 1725.

Kerfoot, K.M., Lavandero, R., Cox, M., Triola, N., Pacinin, C., \& Hanson, D.M. (2006). Conceptual models and the nursing organization. Implementing the AACN synergy model for patient care. Nurse Leader, 2026.

MacPhee, M., Wardrop, A., Campbell, C., \& Wejr, P. (2011). The synergy professional practice model and its patient characteristics tool: A staff empowerment strategy. Nursing Leadership, 24, 4255.

MacPhee, M.K., Jewell, K., Wardrop, A., Ahmed, A., \& Mildon, B. (2010). British Columbia's provincial nursing workload project: Evidence to empowerment. Canadian Journal of Nursing Leadership, 23, 5463.

Pope, B. (2002). The synergy matchup. Nursing Management, 33, 3841. 